\title{
Fracture risk assessment in nursing homes
}

\author{
Nicholas C. Harvey ${ }^{1,2} \cdot$ Cyrus Cooper $^{1,2,3}$
}

Published online: 2 September 2020

(c) European Geriatric Medicine Society 2020

The enormous burden of non-communicable chronic diseases consequent to an increasingly ageing demographic is a clear health priority worldwide, recognised by the World Health Organization in its recent "WHO Report on Ageing and Health" [1]. As populations age, the number of individuals who suffer from non-communicable chronic diseases such as dementia, cardiovascular conditions, osteoporosis and sarcopenia will increase. Using osteoporotic fracture as an example, these demographic changes are projected to result in a doubling in the number of individuals at high fracture risk from around 150 million in 2010 to nearly 300 million in 2040 [2] and the numbers of hip fractures worldwide similarly are expected to rise from 1.66 million in 1990 to 6.26 million annually by 2050 [3]. Osteoporotic fractures cost the EU $€ 39$ billion annually [4] and are associated with substantial morbidity and excess mortality [5]. Frail older persons represent a particularly high risk group, who may require institutional care in residential or nursing homes. This population are known to be at greater risk of fracture than comparable free-living individuals for a variety of reasons, including generally impaired musculoskeletal health resulting in decreased mobility, increased falls risk and lower BMD, with the adjunctive effects of comorbidities such as dementia and cardiovascular disease $[5,6]$.

Whilst this institutionalized population is typically at high fracture risk, there is little consensus about how best to risk stratify individuals within such an environment. Ihama et al., presented in this issue of European Geriatric Medicine, undertook a study of available fracture risk calculators

Cyrus Cooper

cc@mrc.soton.ac.uk

1 MRC Lifecourse Epidemiology Unit, University of Southampton, Southampton General Hospital, Southampton SO16 6YD, UK

2 NIHR Southampton Biomedical Research Centre, University of Southampton and University Hospital Southampton NHS Foundation Trust, Tremona Road, Southampton, UK

3 NIHR Oxford Biomedical Research Centre, University of Oxford, Oxford, UK in a nursing home setting to ascertain their predictive value for the outcomes of falls and fractures [7]. As is not unusual, recruitment presented some difficulties in this old, frail, often demented population, and it is notable that only 217 (35\%) of the 618 residents in 18 homes were enrolled and that of these 217 enrolled, $70 \%$ did not have mental capacity and so consent was obtained from a relative. This of course has implications for the ascertainment of self-reported clinical risk factors and indeed for incident events which were reported by the care home at 12 months. Importantly, there were 325 incident falls amongst the enrolled participants but only 10 fractures. Thus, the statistical power available to investigate the predictive value of the three risk calculators, together with that for the individual clinical risk factors, is modest. Unsurprisingly, the authors did not find that either FRAX, QFracture or the GARVAN calculator were associated with incident falls or fracture events; indeed only BMI and timed up and go test (TUGT) appeared to have predictive value, although these findings must be appreciated in the context of the large number of statistical tests undertaken. An intriguing further question is whether inclusion of age and sex in the models with BMI or TUGT would alter the associations, an important point in the comparison with the three risk calculators since they all include these two attributes as input variables.

The paper from Ihama et al. [7] thus raises important questions about the general approach to risk stratification in frail older people. The issue ultimately is whether we simply take the view that this is a group at very high risk of fracture (the subsequent question then being whether other factors influence the decision to treat with antiosteoporosis medications) or whether we attempt to stratify within this high-risk population. Whilst traditional risk factors for fracture are still highly relevant here, there are other considerations more specific to the nursing home setting, for example greater mobility may be a risk factor for fracture, since there may be a greater risk of falling in this state compared with being bedbound [8]. There may be clear difficulties with the use of oral medications in the setting of dementia, with parenteral therapies such as subcutaneous denosumab providing 
a potential solution. An important consideration is the competing hazard of mortality. Frail older nursing home populations typically have a median survival of around 2 years [9]. Indeed the mortality rate amongst those enrolled to the present study was $25 \%$ over 12 months and these were likely to be the fitter individuals amongst the overall population. There is evidence for a benefit of antiresorptive therapies on vertebral fracture risk at 12 months [10], and over recent years increasing evidence of greater magnitude, and more rapid, effects of anabolic agents, demonstrated head-to-head for risedronate versus teriparatide [11] and alendronate versus romosozumab [12]. Indeed the International Osteoporosis Foundation and the European Society for the Clinical and Economic Aspects of Osteoporosis, Osteoarthritis and Musculoskeletal Diseases have recently released guidance on the targeting of specific therapies by risk level, recommending that those at highest fracture risk should receive anabolic therapies, which will most rapidly reduce their risk of a further fracture [13]. Clearly in the setting of care towards the end-of-life, the likelihood of an event happening relative to the chance of dying from another cause must be taken into account, together with the likelihood of mortality resulting from a fracture [5]. These are difficult considerations in general, and particularly in the specific, and are driven as much by factors such as philosophy and religion as by empirical science.

The issue of fracture versus mortality risk is relevant to the methodology employed in the three risk calculators tested by Ihama et al. QFracture and Garvan calculators are country specific (UK and Australia respectively), generated in single cohorts, and yield an estimate of cumulative fracture risk over a particular time horizon [14-16]. In contrast, FRAX $^{\circledR}$ was derived through meta-analyses of prospective cohort studies from Europe, North America, Asia and Australia including nearly 45,000 individuals, has subsequently been validated in a similar number of patients in other cohorts, and integrates the risk of fracture with risk of death to generate a probability of fracture over a 10 -year period $[16,17]$. It is important to note therefore that the metric of risk generated by FRAX differs from that from QFracture and Garvan calculators and that they cannot be used interchangeably. The metric used is particularly relevant when considering frail older persons, who will have a very high fracture risk (in terms of predicted cumulative incidence) but also a high risk of dying over the time period considered. The QFracture solution to this point is to allow calculation of fracture risk over any time period from 1 to 10 years [14]. The Garvan calculator allows calculation over a 5- or 10 -year time horizon [15]. In using this approach, the assessing physician must make an assumption regarding likely survival time. The problem then is that there is no consensus on what level of cumulative fracture risk constitutes the threshold for antiosteoporosis medication use by different time horizons. One answer might be to multiply up the risk assessed over a shorter time horizon to the equivalent over 10 years. Unfortunately in the case of QFracture, whilst this would allow assessment over a globally established time horizon, there are still no nationally agreed thresholds for intervention on the basis of this particular risk calculator [18]. The question then is whether FRAX probability could or should be subdivided. The critical difference here is that the metric from FRAX, as described above, integrates risk of fracture and risk of death. Thus, for the same cumulative fracture risk, the probability of a fracture over 10 years will be lower if survival is expected to be 5 years compared with 10 years. This approach means that the same metric can be used at all ages as it accommodates the increased risk of death during the time horizon at older ages. Indeed in the "younger old" population, subdivision of the 10-year probability into smaller time periods, for example deriving a 2 -year time horizon by dividing the 10 -year period by five, gives broadly proportionate answers [19]. However, in the oldest old, there can be substantial deviation from 2 years being $1 / 5$ of the 10 -year probability because a fracture cannot occur after the time of predicted death. This problem is even more apparent when considering the effect of a very recent prior fracture on FRAX probability [20].

Taken as a whole, we are still left with the question of whether to consider the institutionalised oldest old as a group generally at high risk of fracture (and then aim to consider the majority for treatment) or whether to attempt to individually risk assess within this population. Given the current global standards for fracture risk assessment, and the linkage of FRAX, but not other risk calculators, to agreed risk thresholds in many settings internationally [13, 21-24], together with the accommodation for competing mortality in the risk model, use of FRAX would seem a reasonable way forward if this were desired. However, its performance characteristics specifically within this population remain to be assessed. For the moment then, particularly given the woefully low proportion of frail older persons, not to mention of the high risk population as a whole, who are appropriately assessed and treated for osteoporosis [25, 26], a pragmatic approach of aiming to treat, unless there are reasons not to, may well be the order of the day.

Acknowledgements We would like to thank the Medical Research Council (UK), National Institute for Health Research, Wellcome Trust, Versus Arthritis, Royal Osteoporosis Society (Osteoporosis and Bone Research Academy) and International Osteoporosis Foundation for supporting this work.

\section{Compliance with ethical standards}

Conflict of interest C. Cooper reports consultancy, lecture fees and honoraria from AMGEN, GSK, Alliance for Better Bone Health, 
MSD, Eli Lilly, Pfizer, Novartis, Servier, Medtronic and Roche outside the scope of the submitted work. N. Harvey reports consultancy, lecture fees and honoraria from Alliance for Better Bone Health, AMGEN, MSD, Eli Lilly, Servier, Shire, UCB, Kyowa Kirin, Consilient Healthcare, Radius Health and Internis Pharma outside the scope of the submitted work.

Ethical approval Not applicable.

Informed consent Not applicable.

\section{References}

1. World Health Organization (2015) WHO world report on ageing and health, Geneva, Switzerland

2. Oden A, McCloskey EV, Kanis JA, Harvey NC, Johansson H (2015) Burden of high fracture probability worldwide: secular increases 2010-2040. Osteoporos Int 26(9):2243-2248

3. Cooper C, Campion G, Melton LJ (1992) Hip fractures in the elderly: a world-wide projection. Osteoporos Int 2(6):285-289

4. Hernlund E, Svedbom A, Ivergard M, Compston J, Cooper C, Stenmark J et al (2013) Osteoporosis in the European Union: medical management, epidemiology and economic burden: a report prepared in collaboration with the International Osteoporosis Foundation (IOF) and the European Federation of Pharmaceutical Industry Associations (EFPIA). Arch Osteoporos 8(1-2):136

5. Harvey N, Dennison E, Cooper C (2010) Osteoporosis: impact on health and economics. Nat Rev Rheumatol 6(2):99-105

6. Rizzoli R, Branco J, Brandi ML, Boonen S, Bruyere O, Cacoub P et al (2014) Management of osteoporosis of the oldest old. Osteoporos Int 25(11):2507-2529

7. Ihama F, Pandyan A, Roffe C (2020) Assessment of fracture risk tools in care home residents: an observational pilot study. Eur Geriat Med 7:s86

8. Chen JS, Simpson JM, March LM, Cameron ID, Cumming RG, Lord SR et al (2008) Risk factors for fracture following a fall among older people in residential care facilities in Australia. $\mathrm{J}$ Am Geriatr Soc 56(11):2020-2026

9. Vossius C, Selbaek G, Saltyte Benth J, Bergh S (2018) Mortality in nursing home residents: a longitudinal study over three years. PLoS ONE 13(9):e0203480

10. Curtis EM, McClung M, Compston JE (2018) Therapeutic approaches to bone protection in adulthood. In: Harvey NC, Cooper C (eds) Osteoporosis: a lifecourse epidemiology approach to skeletal health. CRC Press, Boca Raton, pp 177-192

11. Kendler DL, Marin F, Zerbini CAF, Russo LA, Greenspan SL, Zikan V et al (2018) Effects of teriparatide and risedronate on new fractures in post-menopausal women with severe osteoporosis (VERO): a multicentre, double-blind, double-dummy, randomised controlled trial. Lancet 391(10117):230-240

12. Saag KG, Petersen J, Brandi ML, Karaplis AC, Lorentzon M, Thomas $\mathrm{T}$ et al (2017) Romosozumab or alendronate for fracture prevention in women with osteoporosis. N Engl J Med 377(15):1417-1427
13. Kanis JA, Harvey NC, McCloskey E, Bruyere O, Veronese N, Lorentzon M et al (2020) Algorithm for the management of patients at low, high and very high risk of osteoporotic fractures. Osteoporos Int 31(1):1-12

14. Hippisley-Cox J, Coupland C (2009) Predicting risk of osteoporotic fracture in men and women in England and Wales: prospective derivation and validation of QFractureScores. BMJ 339:b4229

15. Nguyen ND, Frost SA, Center JR, Eisman JA, Nguyen TV (2008) Development of prognostic nomograms for individualizing 5-year and 10-year fracture risks. Osteoporos Int 19(10):1431-1444

16. Kanis JA, Harvey NC, Johansson H, Oden A, McCloskey EV, Leslie WD (2017) Overview of fracture prediction tools. J Clin Densitom 20(3):444-450

17. Assessment of osteoporosis at the primary health-care level (2008) Technical report. WHO Collaborating Centre for Metabolic Bone Diseases, Sheffield

18. Kanis JA, Compston J, Cooper C, Harvey NC, Johansson H, Oden A et al (2016) SIGN Guidelines for Scotland: BMD versus FRAX versus QFracture. Calcif Tissue Int 98(5):417-425

19. Leslie WD, Majumdar SR, Morin SN, Lix LM, Johansson H, Oden A et al (2017) FRAX for fracture prediction shorter and longer than 10 years: the Manitoba BMD registry. Osteoporos Int 28(9):2557-2564

20. Johansson H, Leslie WD, Oden A, Morin SN, Lix LM, Harvey $\mathrm{NC}$ et al (2018) FRAX for fracture prediction over 10 years versus 5 years: the Manitoba BMD registry. Osteoporos Int 29(Suppl 1):S300

21. Kanis JA, Cooper C, Rizzoli R, Reginster JY (2019) European guidance for the diagnosis and management of osteoporosis in postmenopausal women. Osteoporos Int 30(1):3-44

22. Compston J, Cooper A, Cooper C, Gittoes N, Gregson C, Harvey $\mathrm{N}$ et al (2017) UK clinical guideline for the prevention and treatment of osteoporosis. Arch Osteoporos 12(1):43

23. Kanis JA, Harvey NC, Cooper C, Johansson H, Oden A, McCloskey EV (2016) A systematic review of intervention thresholds based on FRAX: a report prepared for the National Osteoporosis Guideline Group and the International Osteoporosis Foundation. Arch Osteoporos 11(1):25

24. Cosman F, de Beur SJ, LeBoff MS, Lewiecki EM, Tanner B, Randall $\mathrm{S}$ et al (2014) Clinician's guide to prevention and treatment of osteoporosis. Osteoporos Int 25(10):2359-2381

25. Harvey NCW, McCloskey EV, Rizzoli R, Kanis JA, Cooper C, Reginster J-Y (2019) Osteoporosis: treatment gaps and health economics. In: Huhtaniemi I, Martini L (eds) Encyclopedia of endocrine diseases, 2nd edn. Academic Press, Oxford, pp 288-295

26. Harvey NC, McCloskey EV, Mitchell PJ, Dawson-Hughes B, Pierroz DD, Reginster JY et al (2017) Mind the (treatment) gap: a global perspective on current and future strategies for prevention of fragility fractures. Osteoporos Int 28(5):1507-1529

Publisher's Note Springer Nature remains neutral with regard to jurisdictional claims in published maps and institutional affiliations. 